\title{
Paramagnetic Meissner Effect and Magnetic Remanence in Granular $\mathrm{MgB}_{2}$
}

\author{
W. A. C. Passos ${ }^{1}$, P. N. Lisboa-Filho ${ }^{1}$, G. L. Fraga ${ }^{2}$, \\ F. W. Fabris ${ }^{2}$, P. Pureur ${ }^{2}$, and W. A. Ortiz ${ }^{1}$ \\ ${ }^{1}$ Grupo de Supercondutividade e Magnetismo, Centro Multidisciplinar para o \\ Desenvolvimento de Materiais Cerâmicos - Departamento de Física, \\ Universidade Federal de São Carlos, Cx.Postal 676, 13565-905 São Carlos, SP, Brazil \\ ${ }^{2}$ Instituto de Física, Universidade Federal do Rio Grande Sul, \\ POB 15051, 91501-970, Porto Alegre, RS, Brazil
}

Received on 28 February, 2002

\begin{abstract}
The magnetic response of $\mathrm{MgB}_{2}$ samples prepared by solid state reaction method, were studied. Important features revealing the granular character of the specimens were verified, as (i) depressed magnetization versus field response, when compared with the corresponding curve for unlinked powder, (ii) the paramagnetic Meissner effect and (iii) the occurrence of a magnetic remanence in a limited interval of temperatures. Experimental evidence is added to indicate that these effects are related to flux pinning.
\end{abstract}

\section{Introduction}

Since the discovery of superconductivity in $\mathrm{MgB}_{2}[1]$, there is an increasing interest on this material, both for fundamental studies and potential applications. As usual, research studies are under way to understand many different aspects of superconductivity in $\mathrm{MgB}_{2}$. Most samples produced thus far present a granular character, similar to that exhibited by ceramic superconductors, displaying a two-component magnetic response.

It has been extensively reported [2-5] that one of the inherent properties of granular superconductors is the paramagnetic Meissner effect (PME), usually identified as a positive magnetization exhibited by certain samples when cooled through the critical temperature in the presence of a magnetic field (field-cooled, FC). As a matter of fact, the PME has been systematically detected when samples of controlled granularity were studied, especially those prepared to behave as tridimensional Josephson junction arrays (JJA) [3-5]. The feature has also appeared on the magnetic response of good-quality thin-films of $\mathrm{Nb}$ and $\mathrm{YBa}_{2} \mathrm{Cu}_{3} \mathrm{O}_{7-\delta \mathrm{g}} \mathrm{YBCO}$ ) cooled in a field perpendicular to the plane of the sample [2]. PME has also been observed in melt-textured YBCO samples submitted to high magnetic fields $(\mathrm{H})[6,7]$.

Another feature of interest is a remanent magnetization (MR), seen in the magnetic response of some specially prepared granular samples as, for instance, dis- ordered tridimensional Josephson arrays of $\mathrm{Nb}[3]$ and YBCO [4]. This feature was also shown to be a characteristic of minimally ordered granular systems $[3,4]$.

\section{Experimental Procedure}

A polycrystalline $\mathrm{MgB}_{2}$ pellet was prepared by solid state reaction. A stoichoimetric mixture of small $\mathrm{Mg}$ chips and fine B powder was wrapped in a Ta foil and sealed under Ar atmosphere in a quartz ampoule. The pellet was sintered at $950{ }^{\circ} \mathrm{C}$ for two hours and furnace cooled to room temperature. Samples for resistivity and magnetic measurements were cutted from the sintered pellet with a diamond saw.

Resistivity $(\rho)$ measurements of the sample up to room temperature were carried out using a lowfrequency $\mathrm{AC}$ technique employing a lock-in amplifier as a null detector. Data obtained at zero applied field are shown in Fig. 1. The inset shows an amplified view of the transition region, evidencing the granular behavior characterized by a double transition.

Magnetic measurements were conducted using two instruments provided by Quantum Design: a 5 Tesla Squid Magnetometer and a 9 Tesla PPMS. Fig. 2 shows the mass magnetization versus field, $\mathrm{M}$ vs. $\mathrm{H}$, curves for two samples at low temperatures: One of the specimens is a small piece $(4.4 \mathrm{mg})$ of a bulk sample, whereas the other consists of powder $(0.7 \mathrm{mg})$ collected during manipulation of the reasonably brittle bulk samples. Both are typical curves of an ordinary type II superconductor. However, comparison of both curves reveals that 
the bulk sample has smaller absolute values of $\mathrm{M}$, indicating that a paramagnetic contribution due to the intergrain fraction is present. Measurements of the magnetic remanence $\left(\mathrm{M}_{\text {rem }}\right)$, taken after application of an AC-field (h), as described in Refs. [2] and [3], are in Fig. 3. The positive remanence appearing in a window of temperatures for certain values of $h$ is a characteristic of a minimally ordered granular systems, similarly to those reported in Refs. [2] and [3] which were prepared to behave as Josephson arrays. The inset of Fig. 3 shows the magnetic response of the same bulk sample submitted to a low AC-field, $h=0.1$ Oe. This curve resembles that of an ordinary superconducting transition, without any trace of MR.

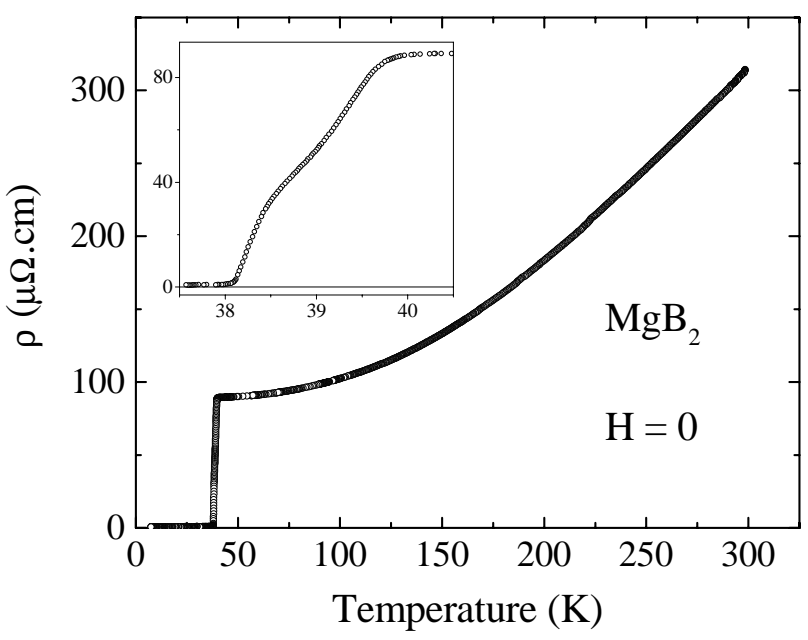

Figure 1. Resistivity measurement of a bulk $\mathrm{MgB}_{2}$ sample: the main graph shows $\rho \times \mathrm{T}$ up to room temperature; inset focuses the double transition, revealing a granular characteristic.

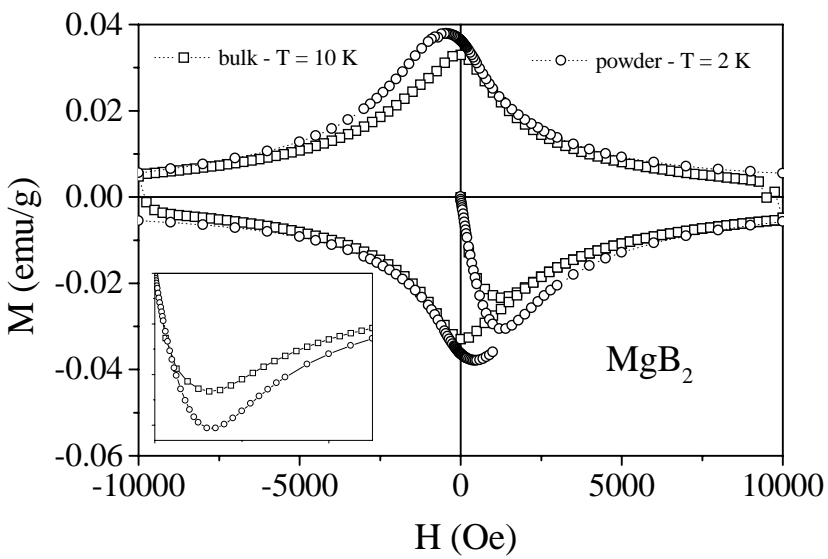

Figure 2. $\mathrm{M} \times \mathrm{H}$ curve for bulk and powder $\mathrm{MgB}_{2}$. Differences between values of powder and bulk are detailed on the inset.

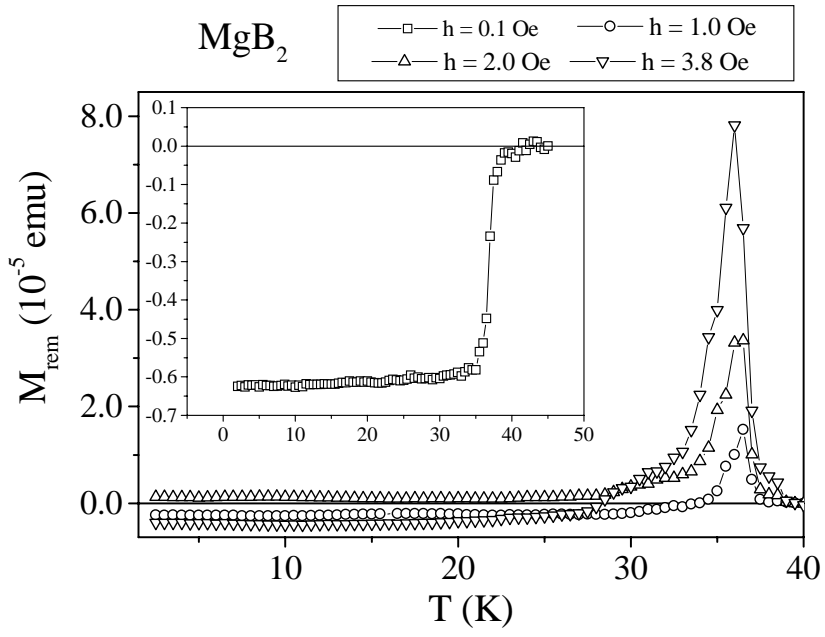

Figure 3. Magnetic remanence of a bulk $\mathrm{MgB}_{2}$ sample after application of an AC field of 0.1, 1.0, 2.0 and 3.8 Oe. Inset shows the measurement for smallest $h$.

Figure 4 shows how the PME is revealed in the studied sample. It is a quite subtle effect, emerging as a difference between the magnetization curves taken in the presence of a magnetic field while cooling (fieldcooled cooling, FCC) or warming (field-cooled warming, FCW) the sample. Interestingly enough, depending on the value of the field the FCW curve lays above or below its corresponding FCC. Curves were selected to evidence this inversion between FCC and FCW magnetizations. Measurements taken at $\mathrm{H}=7000$ Oe are largely amplified in the inset. We have also performed time relaxation measurements of $\mathrm{M}$, which ensured that the effect is not due to relaxation of a metastable state.

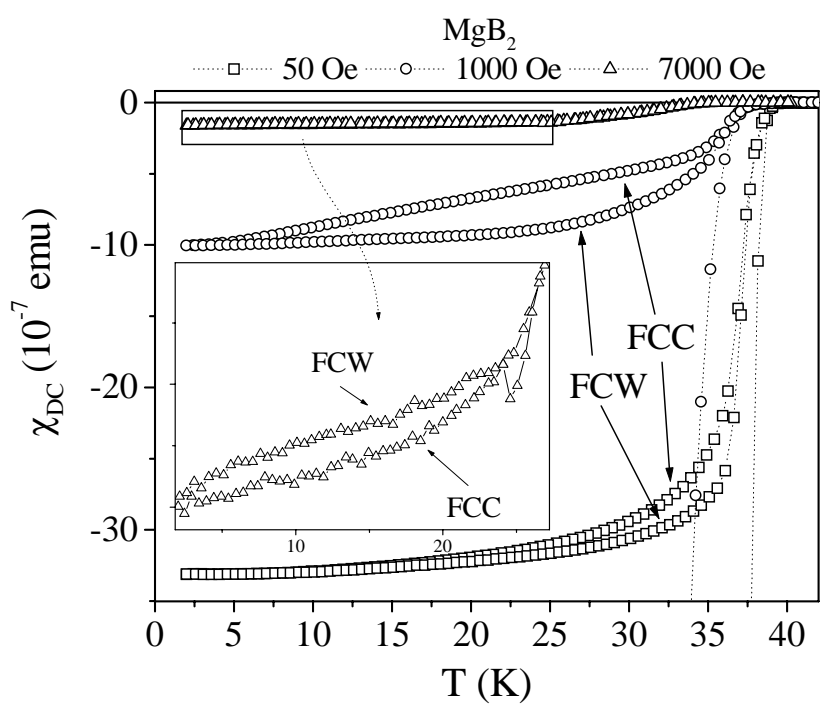

Figure 4. $\chi_{D C}$ of a bulk $\mathrm{MgB}_{2}$ sample exhibiting PME. One can see the inversion of FCW and FCC curves, which is depicted in the inset.

An alternative way to study this peculiar behavior is to scan the temperature up and down in the presence of a field. Fig. 5 presents some of these measurements at fields of 0.1 Tesla and 0.7 Tesla. It is noteworthy 
that these results are independent of the temperature variation rate. Also, regardless of the value of the field, the magnetization always ends at the FCW curve, suggesting that the effect might be related to pinning and that there might be an ideal "training path" for optimizing the magnetic response and, consequently, the critical current of "dirty" specimens of $\mathrm{MgB}_{2}$.

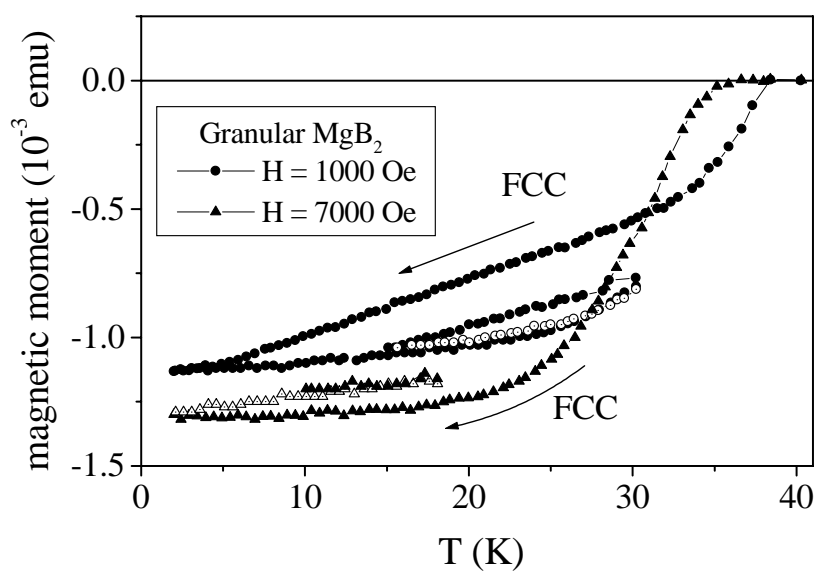

Figure 5. Scans of temperature for a bulk $\mathrm{MgB}_{2}$ sample. The magnetic response suggests a "training path" ending up at the FCW magnetization.

In summing up, we have presented experimental results showing that the studied samples of $\mathrm{MgB}_{2}$ exhibit most of the typical characteristics of a minimally ordered array of weak-links, namely, a suppressed magnetization versus field curve as compared to the corre- sponding powder response, the paramagnetic Meissner effect and a magnetic remanence. Moreover, temperature scans in the presence of an applied field suggest that pinning might be the cause of the observed features, and that the system magnetization always tends to the field-cooled warming branch.

\section{Acknowledgements}

Brazilian financing agencies FAPESP, CNPq, CAPES and FINEP are acknowledged for partial financial support to this work.

\section{References}

[1] J. Nagamatsu, N. Nakagawa, T. Muranaka, Y. Zenitani, and J. Akimitsu, Nature 410, 63 (2001).

[2] W. A. Ortiz P. N. Lisboa-Filho, W. A. C. Passos, and F. M. Araujo-Moreira, Physica C 361, 267 (2001).

[3] W. A. C. Passos, F. M. Araujo-Moreira, and W. A. Ortiz, J. Appl. Phys. 87, 5555 (2000).

[4] W. A. C. Passos, P. N. Lisboa-Filho, and W. A. Ortiz, Physica C 341-348, 2723 (2000).

[5] R. Caparroz, P. N. Lisboa-Filho, W. A. C. Passos, and W. A. Ortiz, Physica C 354, 284 (2001).

[6] F. T. Dias, P. Pureur, P. Rodrigues, and X. Obradors, Physica C 341, 1377 (2000).

[7] F. T. Dias, P. Pureur, P. Rodrigues, and X. Obradors, Physica C 354, 219 (2001). 